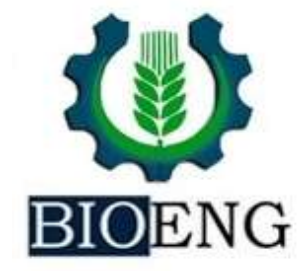

\title{
CRESCIMENTO INICIAL DE CLONES DE EUCALYPTUS EM FUNÇÃO DA ADUBAÇÃO DE NPK
}

\author{
H.A.S. Silva, S. Buzetti, R.N. Gazola ${ }^{1 *}$, G.G. Marques, \\ M.C.M. Teixeira Filho, R.P.D. Gazola
}

UNESP - Universidade Estadual Paulista, Faculdade de Engenharia, Campus de Ilha Solteira, SP, Brasil.

Article history: Received 30 November 2018; Received in revised form 07 February 2019; Accepted 21 February 2019; Available online 30 March 2019.

\section{RESUMO}

A adubação NPK e a escolha do material genético são fundamentais no estabelecimento e na produtividade da cultura. Assim objetivou-se avaliar o efeito de adubação mineral de plantio, no crescimento de clones de eucalipto (Eucalyptus urophylla x Eucalyptus grandis), em solo de Cerrado. O experimento foi conduzido de outubro de 2015 a julho de 2017, na fazenda Campo Verde IV, localizada no município de Selvíria - MS. O delineamento experimental foi o de blocos casualizados com 12 tratamentos e quatro repetições, em esquema fatorial $3 \times 4$, sendo três clones (I144, H13 e 1528) e quatro doses: 0, 160, 320 e $640 \mathrm{~kg} \mathrm{ha}^{-1}$ da fórmula 08$32-16+0,5 \% \mathrm{~B}, 0,5 \% \mathrm{Cu}$ e $0,5 \% \mathrm{Zn}$. Cada parcela foi composta por 42 plantas, distribuídas em sete linhas de seis plantas cada, totalizando $378,0 \mathrm{~m}^{2}$ de área, sendo considerada área útil apenas as seis plantas centrais, totalizando $54,0 \mathrm{~m}^{2}$. Foram avaliados aos 17 meses de idade o diâmetro à altura do peito (DAP) e volume de madeira com casca. A adubação propiciou aumento do DAP e da produtividade de madeira para os três clones, sendo o 1528 mais produtivo.

Palavras-chave: Eucalyptus urophylla $x$ grandis, Cerrado, Volume de madeira.

\section{INITIAL GROWTH OF EUCALYPTUS CLONES IN THE FUNCTION OF NPK FERTILIZATION}

\begin{abstract}
NPK fertilization and the choice of genetic material are fundamental in the establishment and productivity of the crop. The objective of this study was to evaluate the effect of planting mineral fertilization on the growth of eucalyptus urophylla clones (Eucalyptus urophylla $\mathrm{x}$ Eucalyptus grandis) on Cerrado soil. The experiment was conducted from October 2015 to July 2017, at Campo Verde IV farm, located in the municipality of Selvíria - MS. The experimental design was a randomized complete block with 12 treatments and four replications, in a $3 \times 4$ factorial scheme, with three clones (I144, H13 and 1528) and four doses: $0,160,320$ and $640 \mathrm{~kg} \mathrm{ha}^{-1}$ of the formula $08-32-16+0.5 \% \mathrm{~B}, 0.5 \% \mathrm{Cu}$ and $0.5 \% \mathrm{Zn}$. Each plot was composed of 42 plants, distributed in seven rows of six plants each, totaling $378.0 \mathrm{~m}^{2}$ of area, being considered useful area only the six central plants, totaling $54.0 \mathrm{~m}^{2}$. The diameter at breast height (DBH) and volume of wood with bark were evaluated at 17

\footnotetext{
*rngazola@gmail.com

Este trabalho foi apresentado no III Encontro Paulista de Ciência do Solo (III EPCiS), Ilha Solteira, 2018.
} 
months of age. Fertilization increased DBH and wood productivity for the three clones, with the 1528 being more productive.

Keywords: Eucalyptus urophylla $x$ grandis, Cerrado, wood volume.

\section{INTRODUÇÃO}

A silvicultura, em grande expansão no Brasil, coloca o país entre os maiores produtores e exportadores de celulose e papel, e almeja um salto em produtividade. A utilização de técnicas adequadas em eucalipto visa obter plantas vigorosas e produtivas. Nesse sentido, uma das principais ferramentas para alcançar esses objetivos é a escolha adequada da adubação e do tipo de clone de eucalipto.

Entretanto, grande parte do plantio de eucalipto encontra-se em áreas de solo de baixa fertilidade como os da região de Cerrado, o que ocasiona a baixa produtividade da cultura (GAVA et al., 1997).

O eucalipto é uma planta exigente em fósforo $(\mathrm{P})$ principalmente na fase inicial do crescimento, sendo o $\mathrm{P} \quad \mathrm{o}$ nutriente menos acumulado pela cultura (Andrade et al., 2006; Faria et al., 2008). Contudo, de acordo com Faria et al. (2008), o P é um dos limitantes da produção na região do Cerrado. Assim, padronizar a formulação da adubação, já utilizada em grande escala na região em estudo, com o intuito de diminuir os custos de produção, é de fundamental importância.

Devido ao rápido crescimento de plantas de eucalipto no campo ocorre elevada mobilização de nutrientes

\section{MATERIAL E MÉTODOS}

O experimento foi implantado em outubro de 2015 e conduzido até julho de 2017, na Fazenda Campo Verde IV, do grupo Zopone Engenharia, localizada no município de Selvíria-MS, com latitude $20^{\circ} 25^{\prime} \mathrm{S}$ e longitude $51^{\circ} 40^{\prime} \mathrm{O}$ e com altitude de aproximadamente $394 \mathrm{~m}$, região de Três Lagoas-MS. Esta região é caracterizada por clima tropical úmido com estação chuvosa no verão e seca no inverno
(SARTO, 2016). Normalmente, o corte e a colheita da madeira de eucalipto é realizada aos sete anos, sendo conduzido mais um ciclo (a rebrota). Portanto, ocorre muita exportação de nutrientes, o que reduz suas disponibilidades para as plantas, aliado ao fato de que a exportação de nutrientes associada à maioria dos plantios concentra-se em solos de baixa fertilidade natural (SILVEIRA \& MALAVOLTA, 2000).

Segundo Silveira e Malavolta (2000), o teor de $\mathrm{K}$ não é suficiente para atender a demanda das espécies de Eucalyptus em solos florestais, na profundidade de 0,00 0,20 m, sendo que vários trabalhos consultados na literatura relatam resposta do Eucalyptus em solo cujos teores de K estão abaixo de $1,0 \quad \mathrm{mmol}_{\mathrm{c}} \mathrm{dm}^{-3}$ (ALMEIDA et al., 2010; MELO et al., 2016; GAZOLA et al., 2015). A dose adequada de $\mathrm{K}_{2} \mathrm{O}$ em solos com baixo teor de $\mathrm{K}$ deve situar-se na faixa de 120 a 180 $\mathrm{kg} \mathrm{ha}^{-1}$ (SARTO, 2016).

Diante do exposto, objetivou-se avaliar o desenvolvimento inicial, no campo, de mudas de eucalipto de clones (I144, H13 e 1528) comercialmente produzidos e plantados na região de Três Lagoas - MS, avaliando doses de adubo mineral de plantio no desenvolvimento.

- Aw, segundo classificação de Köppen (1948), com pluviosidade, temperatura e umidade relativa do ar média anual de aproximadamente $1330 \mathrm{~mm}, 25^{\circ} \mathrm{C}$ e $66 \%$, respectivamente (CENTURION, 1982).

O delineamento experimental foi o de blocos casualizados com 12 tratamentos e quatro repetições, em esquema fatorial $3 \times 4$, sendo três clones (I144, H13 e 1528 (todos do cruzamento Eucalyptus 
urophylla $x$ Eucalyptus grandis) e quatro doses: $0,160,320$ e $640 \mathrm{~kg} \mathrm{ha}^{-1}$ da fórmula $08-32-16+0,5 \% \mathrm{~B}, 0,5 \% \mathrm{Cu}$ e $0,5 \% \mathrm{Zn}$. O adubo e clones escolhidos foram devido ser os mais utilizados na região pelas grandes empresas de celulose e papel e fazendeiros que plantam eucalipto. E referente as doses escolhidas teve como base a dose da região de $320 \mathrm{~kg} \mathrm{ha}^{-1}$ ser a mais utilizada.

Cada parcela foi composta por 42 plantas, distribuídas em sete linhas de seis plantas cada, totalizando $378,0 \mathrm{~m}^{2}$ de área, sendo considerada como área útil apenas as seis plantas centrais, totalizando $54,0 \mathrm{~m}^{2}$ de área por parcela.

Antecedendo a instalação do experimento, foram coletadas amostras do solo nas profundidades de 0 a 20 e 20 a 40 $\mathrm{cm}$ para determinação dos atributos químicos do solo, segundo a metodologia descrita por Raij et al. (2001). Os atributos químicos na profundidade de 0 a $20 \mathrm{~cm}$ foram: $\mathrm{pH}$ em $\mathrm{CaCl}_{2}$ de 4,3; $12 \mathrm{~g} \mathrm{dm}^{-3} \mathrm{de}$ M.O.; $3 \mathrm{mg} \mathrm{dm}^{-3}$ de $\mathrm{P}$ em resina; e teores de $\mathrm{K}, \mathrm{Ca}, \mathrm{Mg}, \mathrm{H}+\mathrm{Al}$ e $\mathrm{Al}$ de 0,$3 ; 1,0 ; 2,0$; 23,0 e 8,0 $\mathrm{mmol}_{\mathrm{c}} \mathrm{dm}^{-3}$, respectivamente, e a saturação por bases (V) de $11 \%$. Na profundidade de 20 a $40 \mathrm{~cm}$, foram: $\mathrm{pH}$ em $\mathrm{CaCl}_{2}$ de 4,3 e $12 \mathrm{~g} \mathrm{dm}^{-3}$ de M.O.; $2 \mathrm{mg}$ $\mathrm{dm}^{-3}$ de $\mathrm{P}$ em resina; teores de $\mathrm{K}, \mathrm{Ca}, \mathrm{Mg}$, $\mathrm{H}+\mathrm{Al}$ e Al de 0,$3 ; 1,0 ; 1,0 ; 24,0$ e 8,0 $\mathrm{mmol}_{\mathrm{c}} \mathrm{dm}^{-3}$, respectivamente, e V de $9 \%$.

Antes da implantação do experimento foi realizado um conjunto de operações necessárias para implantação da cultura do eucalipto, como: a) controle de formigas: foram aplicados $3 \mathrm{~kg} \mathrm{ha}^{-1}$ de isca formicida granulada sulfluramida, em 21 e 24 de agosto de 2015; c) dessecação da área total (talhão 27): com $3 \mathrm{~kg} \mathrm{ha}^{-1}$ do herbicida glifosato, em 01 de setembro de 2015 e repasse no dia 18 de setembro de 2015 ; d) foi realizada calagem, aplicandose $2.000 \mathrm{~kg} \mathrm{ha}^{-1}$ de calcário dolomítico $($ PRNT $=80 \%)$, dez dias antes do plantio das mudas, em 25 de setembro de 2015, e $1.000 \mathrm{~kg} \mathrm{ha}^{-1}$ de gesso, logo após a calagem, em 28 de setembro de 2015.
O experimento foi instalado no dia 06 de outubro de 2015, iniciando com a subsolagem. Nessa operação fez-se o sulco (com $50 \mathrm{~cm}$ de profundidade na linha de plantio) e a adubação de plantio, utilizando-se a fórmula 08-32-16 + 0,5\% $\mathrm{B}, 0,5 \% \mathrm{Cu}$ e $0,5 \% \mathrm{Zn}$, nas doses de 0 , 160,320 e $640 \mathrm{~kg} \mathrm{ha}^{-1}$.

Antes do plantio, as mudas receberam tratamento, sendo imersas, por alguns segundos, em tanque de aproximadamente $200 \mathrm{~L}$ de água contendo $167 \mathrm{~g}$ de adubo MAP purificado e $100 \mathrm{~g}$ de imidacloprido que é um inseticida sistêmico indicado para o controle de cupins no eucalipto; prática usual na região.

No dia do plantio, 06 de outubro de 2015, foi utilizado gel, polímero hidroretentor, no tanque de irrigação. Esse polímero tem grande capacidade de hidratação da planta, evitando que a água evapore facilmente.

O plantio das mudas foi realizado com espaçamento de 3,60 x 2,50 m, seguido de duas irrigações, sendo uma no dia do plantio e outra três dias após o plantio, para melhor pegamento das mudas. Foi realizado replantio de poucas mudas na área, sendo este desnecessário na área útil.

Para a adubação de cobertura utilizaram-se $180 \mathrm{~kg} \mathrm{ha}^{-1}$ da fórmula 11 $00-22+12 \% \mathrm{~S}$ e $0,8 \% \mathrm{~B}$, em filete contínuo de um lado da planta, em duas adubações, a primeira em 21 de março de 2016 e a segunda em 03 de abril de 2017.

Aos 17 meses após o plantio do eucalipto foram avaliados o diâmetro à altura do peito (DAP), altura e volume de madeira das plantas de eucalipto.

Os dados foram analisados quanto à análise de variância (teste F) e teste de Tukey a $5 \%$ de probabilidade para comparação de médias dos clones e ajustados à regressão polinomial para doses de NPK, utilizando-se do programa SISVAR (FERREIRA, 2008) para análise estatística. 


\section{RESULTADOS E DISCUSSÃO}

Houve efeito quadrático da adubação mineral de plantio para os valores médios de DAP e volume total de madeira com casca, aos 17 meses de idade (Figuras $1 \mathrm{~A}$ e B). O volume total de madeira também foi influenciado pela aplicação das doses de adubação mineral de plantio, com destaque para a dose $320 \mathrm{~kg} \mathrm{ha}^{-1}$ de NPK. Os resultados do volume total da madeira ajustaram-se à função quadrática, sendo o máximo volume estimado com a aplicação de 413, 362 e $465 \mathrm{~kg} \mathrm{ha}^{-1}$ de NPK, para os clones H13, I144 e 1528, respectivamente (Figura 1B).

O mesmo ocorreu com a adubação do Eucalyptus camaldulenses em que o DAP aumentou linearmente aos 16 meses de idade, sendo que a aplicação de $240 \mathrm{~kg}$ $\mathrm{ha}^{-1}$ de $\mathrm{K}_{2} \mathrm{O}$ propiciou maior DAP (COSTA \& TONINI, 2011) e do Eucalyptus urophylla

aos 18 meses de idade, com a aplicação de $180 \mathrm{~kg} \mathrm{ha}^{-1}$ de $\mathrm{K}_{2} \mathrm{O}$ (GAZOLA et al., 2015). Almeida (2009) também verificou que as plantas de E. grandis, quando aplicados $140 \mathrm{~kg} \mathrm{ha}^{-1}$ de $\mathrm{K}_{2} \mathrm{O}$, aos 12 meses de idade, ficaram $54 \%$ mais grossas quando comparadas com a testemunha.O aumento de volume de madeira com casca pode ser observado no crescimento em diâmetro e altura em função das doses de $\mathrm{K}_{2} \mathrm{O}$ (GAZOLA et al., 2015).

Segundo Barros et al. (1990), o nível crítico de $\mathrm{K}$ no solo aumenta com a idade da cultura, em resposta obtida aos 24 meses com a dose mais elevada em relação às avaliações anteriores, e requer maior quantidade desse nutriente durante o ciclo da cultura.

Vários estudos na literatura evidenciaram resposta do eucalitpo a adubação de K (LACLAU et al., 2009; ALMEIDA et al., 2010; MELO et al., 2016; GAZOLA et al., 2015), de N (GAZOLA et al., 2015; PULITO et al., 2015; MELO et al., 2016), de P (GAZOLA et al., 2015; MELO et al., 2016) e de NPK (SILVA et al, 2013).

A maior produtividade de madeira os 17 meses de idade do eucalipto foi obtida para o clone 1528, seguido do I144 e do H13 (menos produtivo) com aplicação de $320 \mathrm{~kg} \mathrm{ha}^{-1}$ da fórmula 08-32-16 + 0,5\% B, $0,5 \% \mathrm{Cu}$ e $0,5 \% \mathrm{Zn}$ no plantio e $360 \mathrm{~kg} \mathrm{ha}^{-}$ ${ }^{1}$ da fórmula $11-00-22+12 \% \mathrm{~S}$ e $0,8 \% \mathrm{~B}$ parcelada em duas aplicaçoes em cobertura, totalizando $130 \mathrm{~kg} \mathrm{ha}^{-1}$ de $\mathrm{K}_{2} \mathrm{O}$ (Figura 1B). Gazola (2014) verificou aos 24 meses de idade do eucalipto I144, maior produtividade de madeira com a aplicação de $151 \mathrm{~kg} \mathrm{ha}^{-1} \mathrm{~K}_{2} \mathrm{O}$.

De acordo com Silva (2011), a recomendação de adubação da literatura precisa ser reavaliada, principalmente devido à utilização de genótipos mais produtivos e provavelmente mais exigentes que os utilizados para gerar a recomendação. Nesse sentido, o clone 1528 foi o menos exigente, pois apresentou maior produtividade de madeira em relação aos outros dois com aplicação da mesma dose de $320 \mathrm{~kg} \mathrm{ha}^{-1}$ da fórmula NPK para os três clones. 

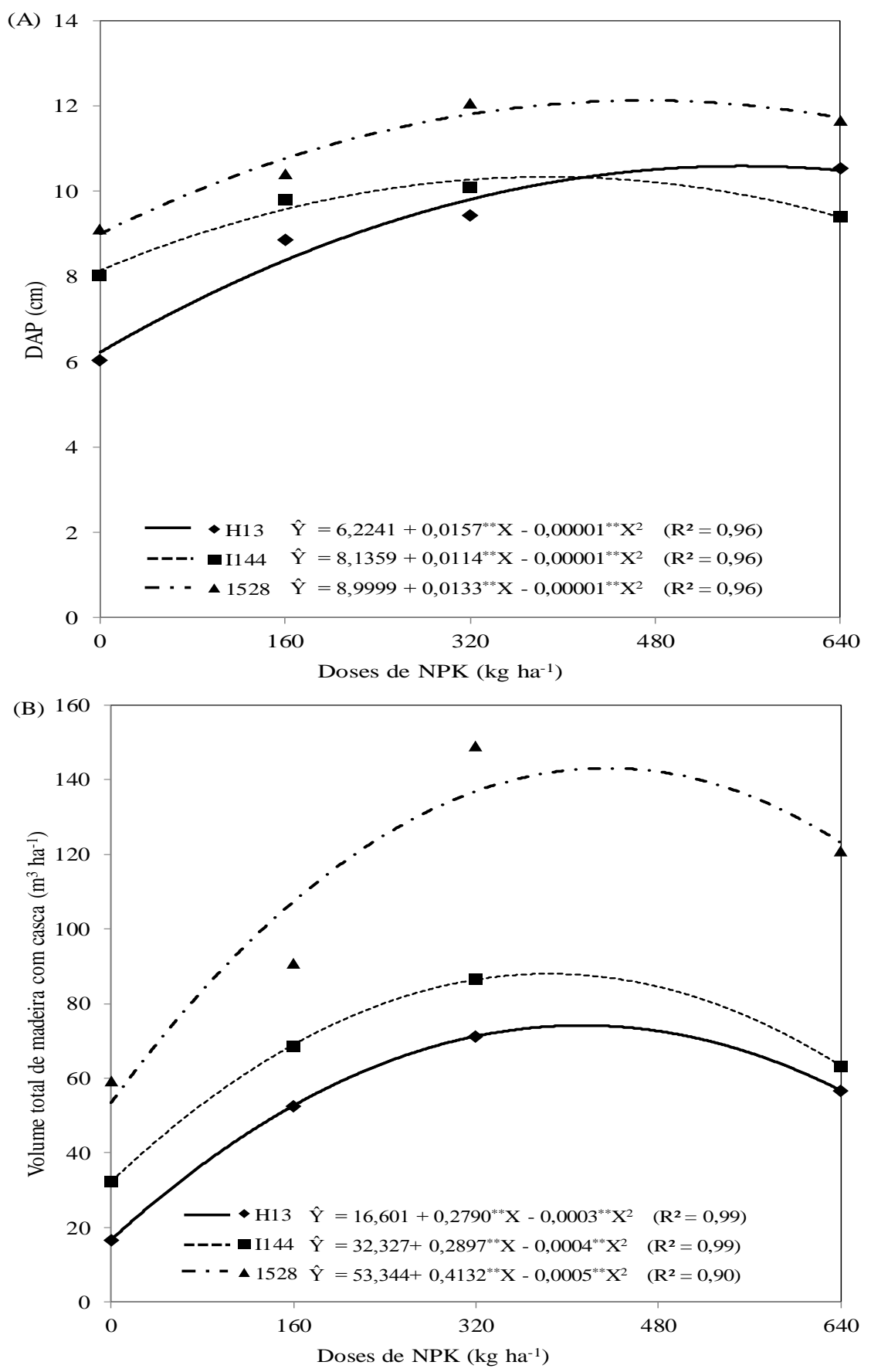

Figura 1. Diâmetro à altura do peito (DAP) e volume total de madeira com casca de clones de eucalipto (A e B, respectivamente) H13, I144 e 1528 aos 17 meses de idade, em função de doses de NPK.

\section{CONCLUSÕES}

O clone 1528 teve comportamento superior em relação aos clones I144 e H13, sendo esse o menos produtivo.

para os três clones foram obtidas com doses superiores a de $320 \mathrm{~kg} \mathrm{ha}^{-1}$ que é a

A maior produtividade de madeira mais comumente utilizada na região.

\section{AGRADECIMENTOS}

Ao viveiro Eucalipto Brasil e viveiro Agroflorestal Terra Flora (grupo Zopone). 


\section{REFERÊNCIAS}

ALMEIDA, J.C.R. Nutrição, crescimento, eficiência de uso de água e de nutrientes em povoamentos de Eucalyptus grandis fertilizados com potássio e sódio. 2009. 112 f. Tese (Doutorado em Recursos Florestais) Escola Superior de Agricultura "Luiz de Queiroz", Universidade de São Paulo, Piracicaba, 2009.

ALMEIDA, J.C.R; LACLAU, J.P.; GONÇALVES, J.L.M.; RANGER， J.; SAINT-ANDRÉ, L. A positive growth response to $\mathrm{NaCl}$ applications in Eucalyptus plantations established on Kdeficient soils. Forest Ecology and Management, n. 259, p. 1786-179, 2010.

ANDRADE， G.C.; BELLOTE， A.F.J.; SILVA, H.D.; RIZZI, N. E.; GAVA, J.L. Acúmulo de nutrientes na biomassa e na serapilheira de Eucalyptus grandis em função da aplicação de lixo urbano e de nutrientes minerais. Boletim de Pesquisa Florestal, n. 53, p. 109-136, 2006.

BARROS, N.F.; NOVAIS, R.F.; NEVES, J.C.L. Fertilização e correção do solo para o plantio de eucalipto. In: BARROS, N.F.; NOVAIS, R.F (Org.). Relação soloeucalipto. Viçosa: Folha de Viçosa, 1990. p. 127-186.

CENTURION, J.F. Balanço hídrico da região de Ilha Solteira. Científica, n. 10, p. 57-61, 1982.

COSTA, M.C.G.; TONINI, H. Efeito de doses de NPK em plantio de Eucalyptus camaldulensis na Savana de Roraima. Boa Vista: Embrapa, 2011. 8 p. Comunicado técnico, 63.

FARIA, G.E.; BARROS, N. F.; CUNHA, V.L.P.; MARTINS, I.S.; MARTINS, R.C.C. Avaliação da produtividade, conteúdo e eficiência de utilização de nutrientes em genótipos de Eucalyptus spp. no Vale do Jequitinhonha, MG. Ciência Florestal, v. 18, n. 3, p. 363-373,
2008.

FERREIRA, D.F. SISVAR: um programa para análises e ensino de estatística. Revista Symposium, v. 6, p. 36-41, 2008.

GAVA， J.L.; GONÇALVES， J.L.M.; SHIBATA, F.Y.; CORRADINI, L. Eficiência relativa de fertilizantes fosfatados no crescimento inicial de eucalipto cultivado em solos do cerrado. Revista Brasileira de Ciência do Solo, v. 21, n. 3, p. 497-504, 1997.

GAZOLA, R.N. Adubação nitrogenada, fosfatada e potássica na cultura do eucalipto (clone I144 - E. urograndis). 2014. 83 f. Dissertação (Mestrado em Agronomia/Sistemas de Produção) Faculdade de Engenharia, Universidade Estadual Paulista, Ilha Solteira, 2014.

GAZOLA, R.N.; BUZETTI, S.; TEIXEIRA FILHO, M.C.M.; DINALLI, R.P.; MORAES, M.L.T.; CELESTRINO, T.S.; SILVA, P.H.M.; DUPAS, E. Doses of $\mathrm{N}, \mathrm{P}$ and $\mathrm{K}$ in the cultivation of eucalyptus in soil originally under Cerrado vegetation. Semina: Ciências Agrárias, n. 35, p. 1895-1912, 2015.

KÖPPEN, W. Climatologia: con un estudio de los climas de la tierra. México: Fondo de Cultura Econômica, 1948. 479 p.

LACLAU, J.P.; ALMEIDA, J.C.R.; GONÇALVES. J.L.M.; SAINT-ANDRÉ, L.; VENTURA, M.; RANGER. J.; MOREIRA, R.M.; NOUVELLON, Y. Influence of nitrogen and potassium fertilization on leaf lifespan and allocation of above-ground growth in Eucalyptus plantations. Tree Physiology, v. 29, n. 1, p. 111-124, 2009.

MELO, E.A.S.C.; GONÇALVES, J.L.M.; ROCHA, J.H.T.; HAKAMADA, R.E.; BAZANI, J.H.; WENZEL, A.V.A.; ARTHUR JUNIOR, J.C.; BORGES, J.S.; 
MALHEIROS, R.; LEMOS, C.C.Z.; FERREIRA, E.V.O.; FERRAZ, A.V. Responses of clonal Eucalypt plantations to $\mathrm{N}, \mathrm{P}$ and $\mathrm{K}$ fertilizer application in different edaphoclimatic conditions. Forests, v. 7, p. 1-15, 2016.

PULITO, A.P.; GONÇALVES, J.L.M.; SMETHURST, P.J.; JUNIOR, J.C.A.; ALCARDE ALVARES, C.; ROCHA, J.H.T.; HÜBNER, A.; DE MORAES, L.F.; MIRANDA, A.C.; KAMOGAWA, M.Y.; ET AL. Available Nitrogen and Responses to Nitrogen Fertilizer in Brazilian Eucalypt Plantations on Soils of Contrasting Texture. Forests, v. 6, p. 973991, 2015.

RAIJ, B.van.; ANDRADE, J.C.; CANTARELLA, H.; QUAGGIO, J.A. Análise química para avaliação da fertilidade de solos tropicais. Campinas: Instituto Agronômico, 2001. 285 p.

SARTO, G.D. Atributos químicos do solo, nutrição e crescimento inicial de brotações de eucalipto em função de doses de potássio. 2016. 49 f. Trabalho de
Conclusão de Curso (Graduação em Agronomia) - Faculdade de Engenharia, Universidade Estadual Paulista, Ilha Solteira, 2016.

SILVA, P.H.M. Impactos das doses e do parcelamento da fertilização na produtividade, lixiviação e ciclagem de nutrientes em plantações de eucalipto. 2011. 118 f. Tese (Doutorado em Ciências/Recursos Florestais) - Escola Superior de Agricultura "Luiz de Queiroz", Universidade de São Paulo, Piracicaba, 2011.

SILVA, P.H.M.; POGGIANI, F.; LIBARDI, P.L.; GONÇALVES, A.N. Fertilizer management of eucalypt plantations on sandy soil in Brazil: Initial growth and nutrient cycling. Forest Ecology and Management, v. 301, p. 6778, 2013.

SILVEIRA, R.L.V.A.; MALAVOLTA, E. Nutrição e adubação potássica em Eucalyptus. Piracicaba: Informações Agronômicas, 2000. 12 p. 